\title{
Microstructural effects and mechanism of bec-hep-bec transformations in polycrystalline iron
}

\author{
Sébastien Merkel $\odot,{ }^{1,2, *}$ Ainhoa Lincot, ${ }^{1,3}$ and Sylvain Petitgirard ${ }^{4, \dagger}$ \\ ${ }^{1}$ Université de Lille, CNRS, INRAE, ENSCL, UMR 8207 - UMET - Unité Matériaux et Transformations, F-59000 Lille, France \\ ${ }^{2}$ Institut Universitaire de France, 75005 Paris, France \\ ${ }^{3}$ Université Grenoble Alpes, Université Savoie Mont-Blanc, CNRS, IRD, IFSTTAR, ISTerre, 38000 Grenoble, France \\ ${ }^{4}$ European Synchrotron Radiation Facility, 38000 Grenoble, France
}

(Received 16 January 2020; revised 2 July 2020; accepted 7 July 2020; published 11 September 2020)

\begin{abstract}
Cycling the $\alpha \leftrightarrow \epsilon$ transformation in polycrystalline $\mathrm{Fe}$ is investigated using in situ x-ray diffraction under quasihydrostatic conditions. The forward $\alpha \rightarrow \epsilon$ transformation starts at $14 \pm 1 \mathrm{GPa}$ and completes at $18 \pm 1 \mathrm{GPa}$ while the reverse $\epsilon \rightarrow \alpha$ transformation starts at $10.5 \pm 0.5 \mathrm{GPa}$ and completes at $6 \pm 1 \mathrm{GPa}$. The anomalous evolution of $c / a$ ratios of $\epsilon$-Fe measured at the onset of the $\alpha \rightarrow \epsilon$ transition in earlier studies is not observed. Microstructural features are consistent with a Burgers path for the transformation. The forward $\alpha \rightarrow \epsilon$ transformation is sensitive to the average and intergranular stresses, inducing a strong variant selection with the $c$ axes of the new $\epsilon$ phase preferentially aligned perpendicular to compression and concentrated into one single orientation. Partial texture memory is observed as the sample returns to the $\alpha$ phase but, as transformation cycles go on, irreversible changes occur in the microstructures that are later fully induced by the cycles of phase transformations.
\end{abstract}

DOI: $10.1103 /$ PhysRevB.102.104103

\section{INTRODUCTION}

Iron is one of the most abundant metals, a widely used technological material, and the main constituent of the earth's core. As such, pressure- and temperature-induced phase transitions in iron have received much attention during the past decades. At $300 \mathrm{~K}$ and about $15 \mathrm{GPa}$, Fe undergoes a bcc $(\alpha-\mathrm{Fe})$ to hcp $(\epsilon-\mathrm{Fe})$ phase transformation [1]. The hysteresis at the transition is large and depends on experimental conditions [2]. As pressure is changed, the transformation occurs rapidly [3], suggesting that it is nondiffusive and martensitic. The structural transition is also associated with changes in magnetic ordering $[4,5]$. Consequently, the $\alpha-\epsilon$ transformation in Fe has been the topic of numerous studies in static highpressure experiments [4-14], dynamic loading experiments [15-19], and numerical models [20-26].

Displacive transformations between bcc and hcp structures in materials other than $\mathrm{Fe}$, such as $\mathrm{Zr}[27,28]$ or $\mathrm{Ti}$ [29-31] follow orientation relationships (ORs) in agreement with the Burgers mechanism [32] (Fig. 1). As for Fe, most static experiments point toward a martensitic mechanism with ORs close to those Burgers $[6,7,10,13]$ although alternative mechanisms have been proposed [9] to explain an anomalous evolution of the $c / a$ ratio of $\epsilon-\mathrm{Fe}$ at the transition. Recent studies, however,

\footnotetext{
*sebastien.merkel@univ-lille.fr
}

$\dagger$ Present address: Department of Earth Sciences, ETH Zürich, Zürich 8092, Switzerland.

Published by the American Physical Society under the terms of the Creative Commons Attribution 4.0 International license. Further distribution of this work must maintain attribution to the author(s) and the published article's title, journal citation, and DOI. including experiments under shock compression [16], static experiments on single crystals [33], and numerical calculations [23,24], all point toward a true Burgers mechanism. The study of Ref. [33], in particular, starting from a single crystal of $\alpha$-Fe, identified the 12 crystalline orientations of $\epsilon$-Fe expected with the Burgers ORs, which validates the Burgers path for the direct transition.

During the bcc to hcp transformation, the Burgers OR establishes 12 potential crystallographic hcp variants from a single bcc orientation (Table I). Conversely, during the hcp to bcc transformation, there are six potential bcc variants from a single hcp orientation (Table II). Hence, transformation cycles could imply a large multiplication of orientation domains and orientations in a polycrystal (Fig. 2). This complexity is of longstanding interest as such phase transformation can induce complex microstructures [34], with effects of history [35], and affect macroscopic properties in materials such as shape memory alloys [36]. Burgers-like transformations, however, do not always produce all the predicted orientations, a process known as texture memory, with important implications regarding bulk materials properties [37]. In situ studies during the $\alpha-\beta-\alpha$ phase transformations in titanium alloys, for instance, demonstrated that variant selection is important in such a case [30]: Upon heating, $\beta$-Ti grows in a preferred direction; upon cooling, a strong selection of $\alpha$ variants occurs, inducing a strong texture memory effect in $\alpha$-Ti after the phase back-transformation.

In Fe, a nearly complete orientation memory was observed during static experiments on single crystals [33]. Whether this memory also applies in polycrystals as well as the microstructures induced by cycles of bcc-hep transformations in polycrystals remain unknown. Microstructures, however, play a critical role in determining the macroscopic behavior of materials. For instance, understanding transformation 

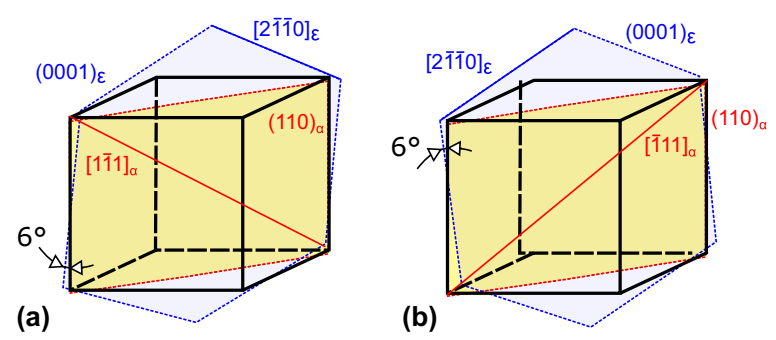

FIG. 1. Schematics of the Burgers orientation relationships. The (110) plane of $\alpha$-Fe transforms to the (0001) plane of $\epsilon$-Fe. The

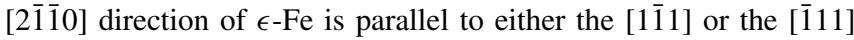
of $\alpha$-Fe (variants $1 \mathrm{a}$ and $1 \mathrm{~b}$ in Table I). Both variants differ by $\approx 12^{\circ}$. There are six equivalent $\{110\}$ planes in $\alpha$-Fe and hence $12 \epsilon$-Fe variants for each $\alpha$-Fe orientation (Table I).

microstructures is the target of the most recent calculations [26,38]. Transformation microstructures are also fundamental to constrain measurements sensitive to grain orientations. In a recent study, for instance, textures induced by the bcc to hcp transformations in Fe were used to evaluate the anisotropy of thermal conductivity in $\mathrm{Fe}$ [39].

Here, we present direct and in situ measurements of microstructures induced during multiple cycles of bcc-hcp phase transformations in polycrystalline $\mathrm{Fe}$. The anomalously large $c / a$ ratio of $\epsilon$-Fe previously reported [9] is not observed. The ORs in $\alpha-\epsilon$ transformation in Fe can be modeled with a Burgers-like mechanism. The forward $\alpha \rightarrow \epsilon$ transformation induces significant variant selection and preferred orientations in $\epsilon$-Fe. The back-transformation to $\alpha$-Fe displays a partial memory effect. Overall, the starting microstructures are progressively erased with increasing transformation cycles.

\section{METHODS}

Two experiments were performed at the ID27 beamline of the European Synchrotron Radiation Facility. In both cases, the starting material was a commercial Fe foil, $25-\mu \mathrm{m}$ thick in run 1 - and $5-\mu \mathrm{m}$ thick in run 2 . The starting materials were

TABLE I. Crystallographic orientation relationships for the 12 variants of the bcc to hcp transformation in the Burgers mechanism. Variants based on the same bcc planes have been grouped in pairs (e.g., V1.a V1.b) as their final orientation differs by only $\approx 12^{\circ}$. The orientations of the hcp variants are represented in Fig. 8.

\begin{tabular}{lccc}
\hline \hline & Planes & Directions & bcc $\rightarrow$ hcp \\
\hline V1 & $(110)_{\mathrm{bcc}} \|(0001)_{\mathrm{hcp}}$ & {$[1 \overline{1} 1]_{\mathrm{bcc}} \|[2 \overline{1} \overline{1} 0]_{\mathrm{hcp}}$} & V1.a \\
& & {$[\overline{1} 11]_{\mathrm{bcc}} \|[2 \overline{1} \overline{1} 0]_{\mathrm{hcp}}$} & V1.b \\
V2 & $(\overline{1} 10)_{\mathrm{bcc}} \|(0001)_{\mathrm{hcp}}$ & {$[11 \overline{1}]_{\mathrm{bcc}} \|[2 \overline{1} \overline{1} 0]_{\mathrm{hcp}}$} & V2.a \\
& & {$[111]_{\mathrm{bcc}} \|[2 \overline{1} \overline{1} 0]_{\mathrm{hcp}}$} & V2.b \\
V3 & $(101)_{\mathrm{bcc}} \|(0001)_{\mathrm{hcp}}$ & {$[11 \overline{1}]_{\mathrm{bcc}} \|[2 \overline{1} \overline{1} 0]_{\mathrm{hcp}}$} & V3.a \\
& & {$[1 \overline{1} \overline{1}]_{\mathrm{bcc}} \|[2 \overline{1} \overline{1} 0]_{\mathrm{hcp}}$} & V3.b \\
V4 & $(011)_{\mathrm{bcc}} \|(0001)_{\mathrm{hcp}}$ & {$[11 \overline{1}]_{\mathrm{bcc}} \|[2 \overline{1} \overline{1} 0]_{\mathrm{hcp}}$} & V4.a \\
& & {$[1 \overline{1} 1]_{\mathrm{bcc}} \|[2 \overline{1} \overline{1} 0]_{\mathrm{hcp}}$} & V4.b \\
V5 & $(\overline{1} 01)_{\mathrm{bcc}} \|(0001)_{\mathrm{hcp}}$ & {$[1 \overline{1} 1]_{\mathrm{bcc}} \|[2 \overline{1} \overline{1} 0]_{\mathrm{hcp}}$} & V5.a \\
& & {$[111]_{\mathrm{bcc}} \|[2 \overline{1} \overline{1} 0]_{\mathrm{hcp}}$} & V5.b \\
V6 & $(0 \overline{1} 1)_{\mathrm{bcc}} \|(0001)_{\mathrm{hcp}}$ & {$[\overline{1} 11]_{\mathrm{bcc}} \|[2 \overline{1} \overline{1} 0]_{\mathrm{hcp}}$} & V6.a \\
& & {$[111]_{\mathrm{bcc}} \|[2 \overline{1} \overline{1} 0]_{\mathrm{hcp}}$} & V6.b \\
\hline \hline
\end{tabular}

TABLE II. Crystallographic orientation relationships for the six variants of the hcp to bcc transformation in the Burgers mechanism. The orientations of the bcc variants are represented in Fig. 9.

\begin{tabular}{llc}
\hline \hline hcp $\rightarrow$ bcc & \multicolumn{1}{c}{ Planes } & Directions \\
\hline V1 & $(0001)_{\mathrm{hcp}} \|(1 \overline{1} 0)_{\mathrm{bcc}}$ & {$[2 \overline{1} \overline{1} 0]_{\mathrm{hcp}} \|[111]_{\mathrm{bcc}}$} \\
V2 & $(0001)_{\mathrm{hcp}} \|(110)_{\mathrm{bcc}}$ & {$[2 \overline{1} \overline{1} 0]_{\mathrm{hcp}} \|[1 \overline{1} 1]_{\mathrm{bcc}}$} \\
V3 & $(0001)_{\mathrm{hcp}} \|(1 \overline{1} 0)_{\mathrm{bcc}}$ & {$[11 \overline{2} 0]_{\mathrm{hcp}} \|[111]_{\mathrm{bcc}}$} \\
V4 & $(0001)_{\mathrm{hcp}} \|(110)_{\mathrm{bcc}}$ & {$[11 \overline{\bar{L}} 0]_{\mathrm{hcp}} \|[1 \overline{1} 1]_{\mathrm{bcc}}$} \\
V5 & $(0001)_{\mathrm{hcp}} \|(1 \overline{1} 0)_{\mathrm{bcc}}$ & {$[\overline{1} 2 \overline{1} 0]_{\mathrm{hcp}} \|[111]_{\mathrm{bcc}}$} \\
V6 & $(0001)_{\mathrm{hcp}} \|(110)_{\mathrm{bcc}}$ & {$[\overline{1} 2 \overline{1} 0]_{\mathrm{hcp}} \|[1 \overline{1} 1]_{\mathrm{bcc}}$} \\
\hline \hline
\end{tabular}

textured, due to the fabrication of the foils, and this starting texture is used as a reference for polycrystal orientations. Foils were loaded in diamond anvil cells equipped with $300-\mu \mathrm{m}$ culet diamonds and rhenium gaskets preindented to $40-\mu \mathrm{m}$ thickness, along with ruby spheres as pressure calibrants. In both runs, and to maintain the lowest levels of nonhydrostatic stress, we used helium as a pressure medium for which differential stress should not exceed $0.1 \mathrm{GPa}$ in the current pressure range [40].

In both runs, samples were driven over three pressure cycles using a remote-controlled pressure drive, up to $\sim 25 \mathrm{GPa}$, above the $\alpha$ - to $\epsilon$-Fe transformation, and down to $\sim 5 \mathrm{GPa}$, below the $\epsilon$ - to $\alpha$-Fe transformation [2]. Each cycle lasted several hours to avoid effects of kinetics, damaging the rhenium gaskets, and minimizing nonhydrostatic stress in the sample. After each pressure change, and after pressure stabilization, angular-dispersive $\mathrm{x}$-ray diffraction data were collected using an incident $\mathrm{x}$-ray beam of $8 \mathrm{x} 9 \mu \mathrm{m}^{2}$ in run 1 and of $2 \times 3 \mu \mathrm{m}^{2}$ in run 2 . Wavelength was set to $0.3738 \AA$ and diffraction data collected on a Mar CCD placed at $206.124 \mathrm{~mm}$ and $205.479 \mathrm{~mm}$ from the sample in runs 1 and 2, respectively [Fig. 3(a)].

The diffraction data shows well-defined peaks of $\alpha$ - or $\epsilon$-Fe [Fig. 3(c)] with significant variations of diffraction intensity with orientation, indicative of the sample texture. The data was processed using the Rietveld refinement software MAUD [41], using procedures described previously [42]. MAUD allows for the extraction of the volume fractions of $\alpha$ - and $\epsilon$-Fe, lattice parameters, and microstructures for each phase. For high-symmetry materials with redundant texture information, the E-WIMV algorithm in MAUD allows extracting a full texture from a single diffraction image. The convergence of

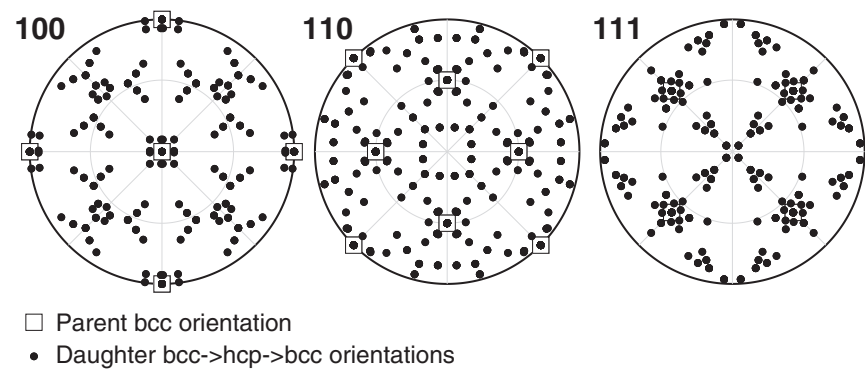

FIG. 2. Potential orientations of $\alpha$-Fe crystals after one cycle of bcc $\rightarrow$ hcp $\rightarrow$ bcc transformations. The parent and daughter $\alpha$-Fe orientation are shown with open squares and dark disks, respectively. 


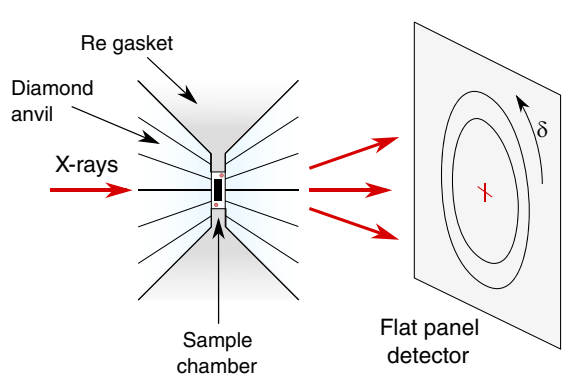

(a) (b)

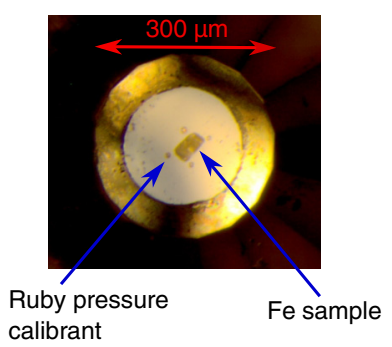

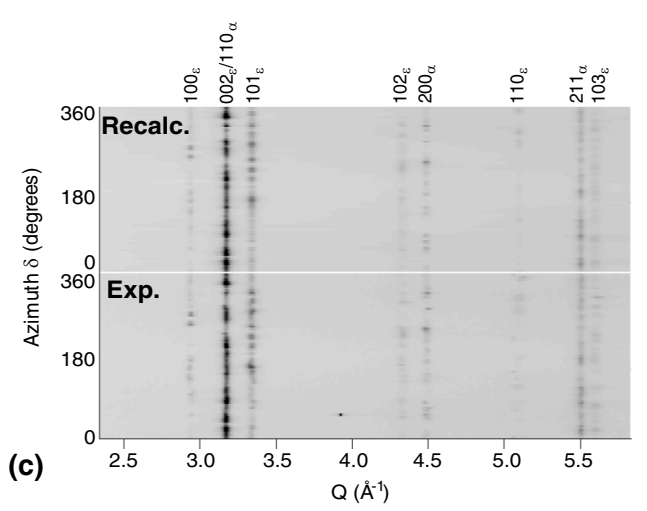

FIG. 3. (a) Experimental setup. The sample chamber is drilled in a rhenium gasket and compressed between two diamond anvils. The Fe sample (black) is confined along with a Ruby ball (red) inside a helium pressure medium (white). X-ray diffraction is collected in angledispersive geometry with the incident x-ray beam parallel to the diamonds compression axis. (b) Picture of the sample loading for run 2. (c) Diffraction image of a sample with $50 \%$ of $\alpha$-Fe and $50 \%$ of $\epsilon$-Fe collected at $15.4 \mathrm{GPa}$ in Exp. 1 (bottom) with the image recalculated after Rietveld refinement in MAUD (top). All diffraction peaks are from the Fe sample. Texture is evident as intensity variations along diffraction peaks. The close similarity between experimental and recalculated images indicates an excellent fit.

such a texture fit with poor pole figure coverage is possible thanks to the tube projection in the E-WIMV algorithm [43], which was successfully tested on materials with the hcp structure [44]. Finally, the obtained textures were further smoothed using a $7.5^{\circ}$ Gaussian filter to remove artifacts from the fitting procedures.

\section{EXPERIMENTAL RESULTS}

\section{A. Phase transformation hysteresis}

The onset of the forward $\alpha \rightarrow \epsilon$ transition is found at $14 \pm 1 \mathrm{GPa}$ and it is completed by $18 \pm 1 \mathrm{GPa}$. The reverse transformation starts at $10.5 \pm 0.5 \mathrm{GPa}$ and finishes at $6 \pm 1 \mathrm{GPa}$ (Fig. 4). The hysteresis and phase coexistence pressure ranges are reproducible and not affected by further pressure cycling. A small proportion of $\epsilon$-Fe and $\alpha$-Fe does persist above $20 \mathrm{GPa}$ and below $4.5 \mathrm{GPa}$, respectively, in agreement with results from the literature [8].

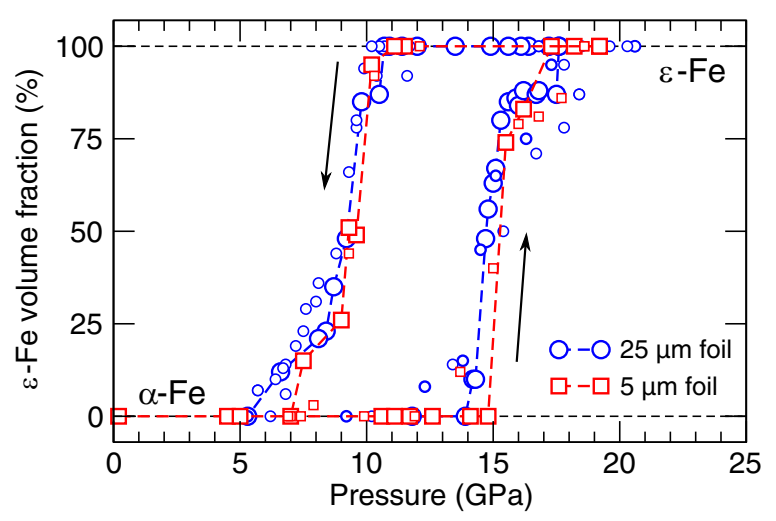

FIG. 4. Volume fraction of $\epsilon$-Fe vs pressure for runs 1 (blue circles) and 2 (red squares). Larger symbols are data from the first cycle of compression and decompression. Smaller symbols are data collected upon further cycles of compression and decompression. Raw data for this figure is available in the Supplemental Material [54].

\section{B. Average domain sizes}

Rietveld refinement allows for extracting the average coherent crystallographic domain size. Accordingly, the average domain size in the starting $\alpha$-Fe sample is $\approx 400 \AA$ in Exp. 1 and $550 \AA$ in Exp. 2 (Fig. 5). After transformation, the average domain sizes in $\epsilon-\mathrm{Fe}$ are $\approx 200 \AA$ is Exp. 1 and $300 \AA$ in Exp. 2. Interestingly, the average domain sizes measured in $\alpha$ - and $\epsilon$-Fe are nearly independent of the phase transformation cycles. As $\epsilon$-Fe back-transforms into $\alpha$-Fe, the average domain size increases back to values slightly below those measured at the start of the experiment. Along the same line, the average domain size in $\epsilon$-Fe is independent of the number of phase transformation cycles.

\section{C. $\mathrm{c} / \mathrm{a}$ ratio of $\epsilon-\mathrm{Fe}$}

A linear regression on the $c / a$ ratio of $\epsilon$-Fe measured in this work leads to $c / a=1.613(1)-0.00039(7) * P$, where $P$ is expressed in GPa and numbers in parenthesis are standard errors on the last digit (Fig. 6). This fit is in agreement with recent static studies starting from a single crystal of $\alpha$-Fe [33], powdered $\alpha-\mathrm{Fe}$ [45], as well as in situ measurements in shock-compressed polycrystalline $\alpha$-Fe [46]. There are small deviations from the fit when the phase fraction of $\epsilon$-Fe is low (i.e., below $15 \mathrm{GPa}$ upon compression and below $10 \mathrm{GPa}$ upon decompression). Large variations of the $c / a$ ratio of $\epsilon-\mathrm{Fe}$ at low pressures measured in earlier studies [6,7,9] are not observed.

\section{Texture in $\epsilon$-Fe}

Figure 7 presents the texture measured in $\alpha$ - and $\epsilon$-Fe at the end of each cycle of phase transformation. The texture measured in $\alpha-\mathrm{Fe}$ at the start of the experiment will be used as a reference for the rest of the analysis. Textures measured in $\epsilon$-Fe at cycles 1,2 , and 3 are results of the phase transformation from the previous $\alpha$-Fe polycrystal. Textures measured in $\alpha$-Fe after cycles 1, 2, and 3 are results of the phase transformation from the previous $\epsilon$-Fe polycrystal. 

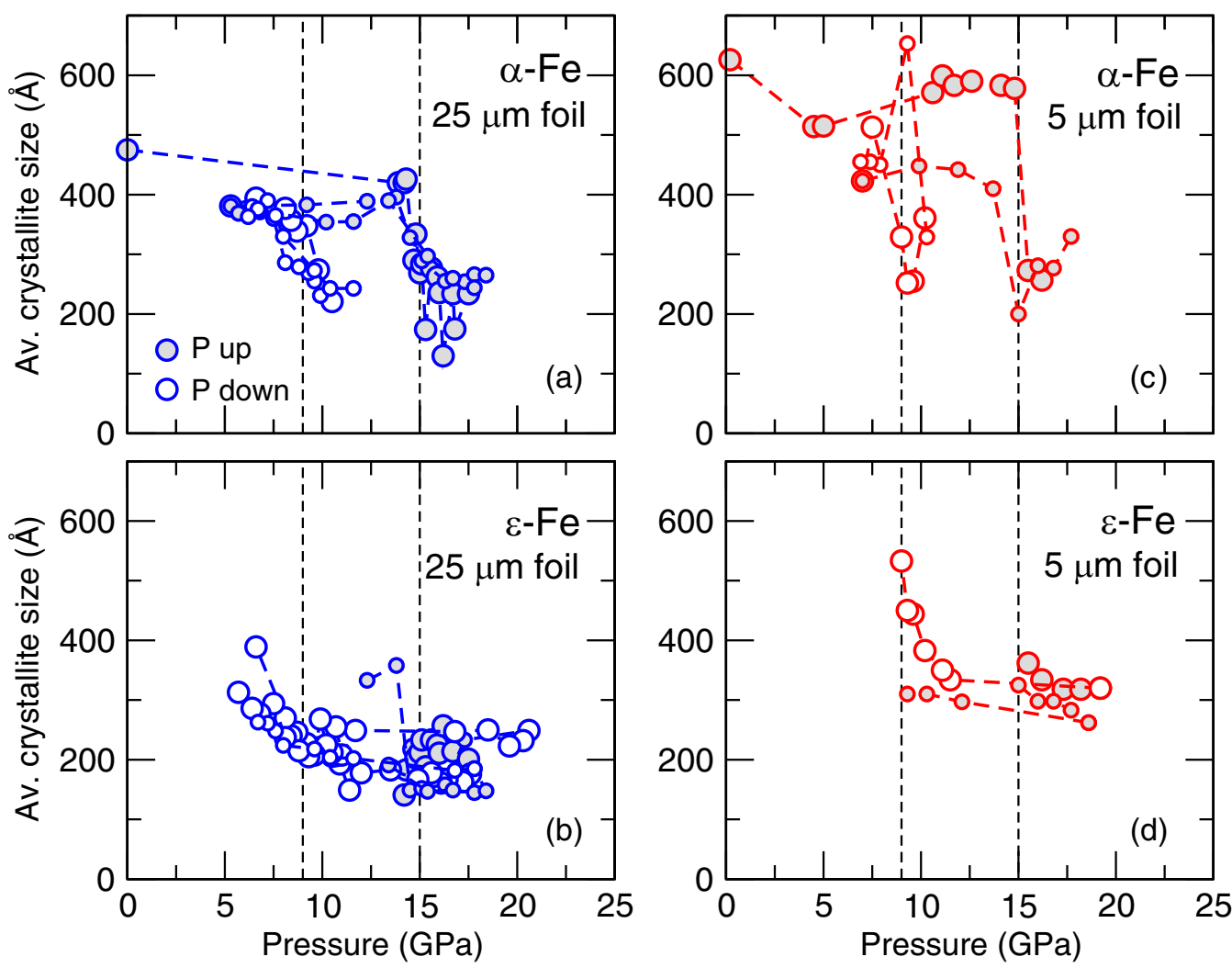

FIG. 5. Average domain size for $\alpha$ - (a), (c), and $\epsilon$-Fe (b), (d) measured in runs 1 (a), (b) and 2 (c), (d). Larger symbols are data from the first cycle of compression and decompression. Smaller symbols indicate data collected on further pressure cycles. Grey and white filled symbols indicate data upon increasing and decreasing pressures, respectively. Raw data for this figure is available in the Supplemental Material [54].

After the first $\alpha \rightarrow \epsilon$ transformation, we observe strong preferred orientations for $\epsilon$-Fe, with the $c$ axes preferentially aligned perpendicular to the compression direction. Furthermore, the $c$ axes of the $\epsilon$-Fe polycrystals tend to concentrate in a given direction perpendicular to compression. This is observed in both experiments, although the directions in which $c$ axes concentrate perpendicular to the compression is sample dependent.

This transformation texture strengthens upon further cycles of $\alpha \leftrightarrow \epsilon$ transformations, with an even stronger alignment of the $c$ axes in a given direction perpendicular to the compression. The direction in which $c$ axes concentrate does not evolve upon cycling $\alpha \leftrightarrow \epsilon$ phase transformations. The cycles of transformations between $\alpha$ - and $\epsilon$-Fe strengthens the transformation texture but does not affect its geometrical features.

\section{E. Texture in $\alpha$-Fe}

The starting texture in $\alpha$-Fe results from the manufacturing process of the foils used as a starting material. One can also notice that the starting texture in Exp. 2 (using a 5- $\mu \mathrm{m}$ thick sheet) is much stronger than that in Exp. 1 (using a $25-\mu \mathrm{m}$-thick sheet). Indeed, the fabrication of a $5-\mu \mathrm{m}$ sheet of $\mathrm{Fe}$ involves more deformation of the original $\mathrm{Fe}$ block than for $25 \mu \mathrm{m}$. At the end of the first cycle of $\alpha \leftrightarrow \epsilon$ phase transformations, a memory remains from the original $\alpha$-Fe texture but new features, inherited from the $\alpha \rightarrow \epsilon \rightarrow \alpha$ transformation, appear in the figure.
Upon further pressure cycling, new features of the $\alpha \rightarrow$ $\epsilon \rightarrow \alpha$ transformation increase in intensity, gradually erasing the memory of the original texture. At the end of the experiment for Exp. 1, the texture $\alpha$-Fe texture bears little resemblance with that measured at the start of the experiment. For Exp. 2, the strong concentration of 100 axes parallel to compression is preserved. The 110 projection, however, is very different from that observed at the start of the experiment.

\section{TRANSFORMATION TEXTURE SIMULATIONS}

\section{A. Phase transformation modeling}

Textures induced by martensitic transformations can be modeled using sets of ORs between a parent and a daughter phase. Four ORs are regularly reported in the literature for relating bcc and hcp phase orientations [47,48]: The classical Burgers OR [32], as well as others such as the Pitsch-Schrader [49], Potter [50], and Rong-Dunlop [51] ORs (Table III). Early publications on the $\alpha-\epsilon$ transformation in Fe reported ORs similar to those of Pitsch-Schrader [6,7] as well as more complex mechanisms [9]. Recent publications, however, demonstrated that the Burgers orientation relationships apply for the $\alpha \rightarrow \epsilon$ transformation in Fe under static conditions $[13,33]$.

Whatever the exact OR, however, the multiple $\alpha \rightarrow \epsilon \rightarrow \alpha$ transformation cycles should lead to a large multiplication of orientation variants in the polycrystal (Fig. 2). This 
TABLE III. Commonly observed orientation relationships (ORs) between bcc and hcp phases: Pitsch-Schrader [49], Burgers [32], Potter [50], and Rong-Dunlop [51]. The Pitsch-Schrader ORs are identical to those predicted by the mechanism of Mao, Bassett, and Takahashi for the $\alpha$ to $\epsilon$ transformation in Fe [6,7].

\begin{tabular}{lccc}
\hline \hline Pitsch-Schrader & Burgers & Potter & Rong-Dunlop \\
\hline$(0001)_{\mathrm{hcp}} / /\{011\}_{\mathrm{bcc}}$ & $(0001)_{\mathrm{hcp}} / /\{011\}_{\mathrm{bcc}}$ & $(0001)_{\mathrm{hcp}} 2^{\circ}$ from $\{011\}_{\mathrm{bcc}}$ & $(0001)_{\mathrm{hcp}} / /\{021\}_{\mathrm{bcc}}$ \\
$\{1 \overline{1} 00\}_{\mathrm{hcp}} / /\{0 \overline{1} 1\}_{\mathrm{bcc}}$ & $\{1 \overline{1} 00\}_{\mathrm{hcp}} / /\{121\}_{\mathrm{bcc}}$ & $\{1 \overline{1} 01\}_{\mathrm{hcp}} / /\{110\}_{\mathrm{bcc}}$ & $\{1 \overline{1} 00\}_{\mathrm{hcp}} / /\{0 \overline{1} 2\}_{\mathrm{bcc}}$ \\
$\langle 11 \overline{2} 0\rangle_{\mathrm{hcp}} / /\langle 100\rangle_{\mathrm{bcc}}$ & $\langle 11 \overline{2} 0\rangle_{\mathrm{hcp}} / /\langle 1 \overline{1} 1\rangle_{\mathrm{bcc}}$ & $\langle 11 \overline{2} 0\rangle_{\mathrm{hcp}} / /\langle 1 \overline{1} 1\rangle_{\mathrm{bcc}}$ & $\langle 11 \overline{1} 0\rangle_{\mathrm{hcp}} / /\langle 100\rangle_{\mathrm{bcc}}$ \\
\hline \hline
\end{tabular}

prediction is incompatible with the texture strengthening and texture memory observed in $\epsilon$ - and $\alpha$-Fe, respectively (Fig. 7). It is also incompatible with the consistent average domain sizes measured at all cycles of phase transformations (Fig. 5).

To understand this behavior, the transformations are modeled based on the Burgers OR for the $\alpha \rightarrow \epsilon$ (Table I, Fig. 8) and $\epsilon \rightarrow \alpha$ (Table II, Fig. 9) transformation, investigating the effect of variant selection and memory in the transformation. In all cases, the experimental texture prior to the phase transformation is converted to a set of 3000 weighted orientations representing the experimentally measured texture. We then apply the Burgers OR, with various levels of variant selection to reproduce the experimentally measured texture after phase transformation. Details are provided below.

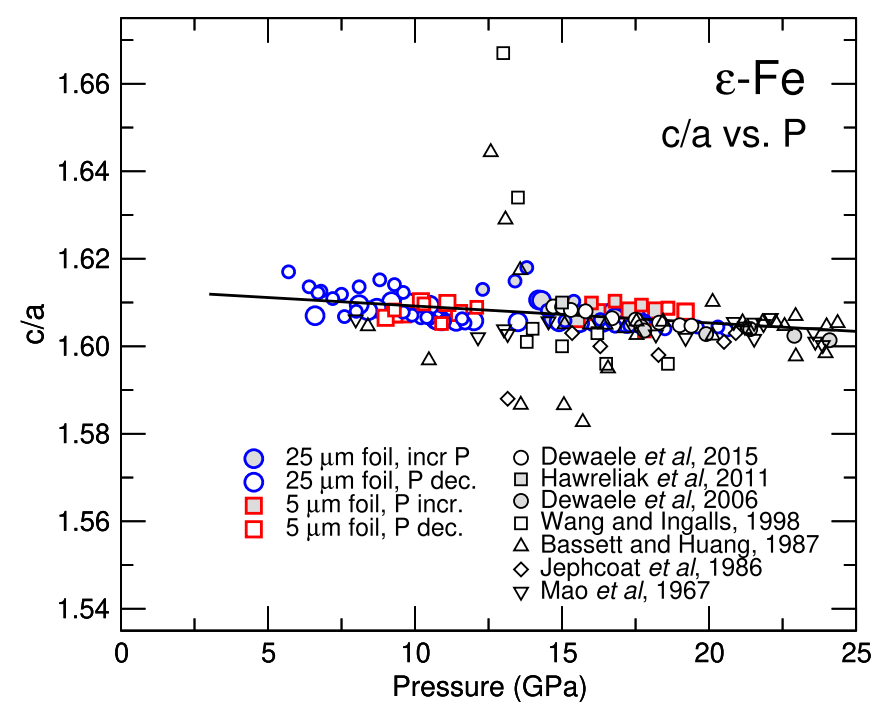

FIG. 6. c/a ratio for $\epsilon$-Fe measured in runs 1 (blue circles) and 2 (red squares). Larger symbols are data from the first cycle of compression and decompression. Smaller symbols indicate data collected upon further cycles of compression and decompression. Gray and white filled symbols indicate data upon increasing and decreasing pressures, respectively. The figure also includes diamond anvil cell data from Refs. [33] (white-filled black circles), [45] (grey-filled black circles), [9] (white-filled black squares), [7] (upward triangles), [55] (black diamonds), and [6] (downward triangles), as well as the shock compression data of Ref. [46] (gray-filled black squares). Solid line is a linear regression to the data from the current study. Raw data for this figure is available in the Supplemental Material [54].

\section{B. $\alpha$ to $\epsilon$ transformation}

First, all transformation variants (Table I) are considered with an equal probability. Each orientation of $\alpha$-Fe gives birth to 12 daughter orientations in $\epsilon$-Fe, resulting in a texture based on 36000 orientations. The simulated textures in $\epsilon$-Fe are far weaker than observed experimentally (Fig. 10) and do not match the concentration of $c$ axes perpendicular to compression observed experimentally.

For each orientation in the parent bcc texture, we hence apply the transformation selecting variants with their $c$ axes perpendicular to the compression direction, within $30^{\circ}$. If no such variant is found, we allow equal probability for the 12 transformation variants. As expected, $c$ axes of the resulting hcp polycrystal lie perpendicular to compression, as observed in the experiment. The strong concentration of $c$ axes observed experimentally around a single orientation, however, is not reproduced.

Next, we impose a second selection rule, selecting variants that agree with two conditions: (i) that the $c$ axis of $\epsilon$-Fe is perpendicular to the compression direction, within $30^{\circ}$, and (ii) that the $c$ axis lies in the same quadrant of the pole figure as in the experiment. Again, we allow equal probability for the 12 transformation variants if no such variant is found. The simulations then reproduces the main features observed in the experiment, albeit sometimes with a stronger texture strength than in the experiment.

Finally, to match the experimental and simulated texture strength, we reduce the effect of variant selection by imposing a ratio of grains that will undergo the transformation through any random variant. For Exp. 1, with a $25-\mu \mathrm{m}$-thick foil, the ratio of grains undergoing the transformation through a random variant is $20 \%, 7 \%$, and $2 \%$ for cycles 1,2 , and 3 , respectively. For Exp. 2, with 5- $\mu$ m-thick foil, we find that $5 \%$ random variant selection is reasonable for all cycles.

\section{C. $\epsilon$ to $\alpha$ transformation}

Simulations of the effect of Burgers OR for textures in $\alpha$-Fe after transformation from $\epsilon$-Fe are shown in Fig. 9 . The simulated textures share common features with those observed experimentally, particularly in the 110 pole figures.

The experimental textures, however, show a memory from the original textures, before transformation into the hcp phase, such as the maximum at the center of the 100 pole figure. The experimental textures in $\alpha$-Fe hence lie between those simulated with the Burgers mechanism and those of $\alpha$-Fe in the previous cycle. We hence observe a memory of the initial $\alpha$-Fe microstructure. 
(a)

Exp. 1

bcc-Fe foil

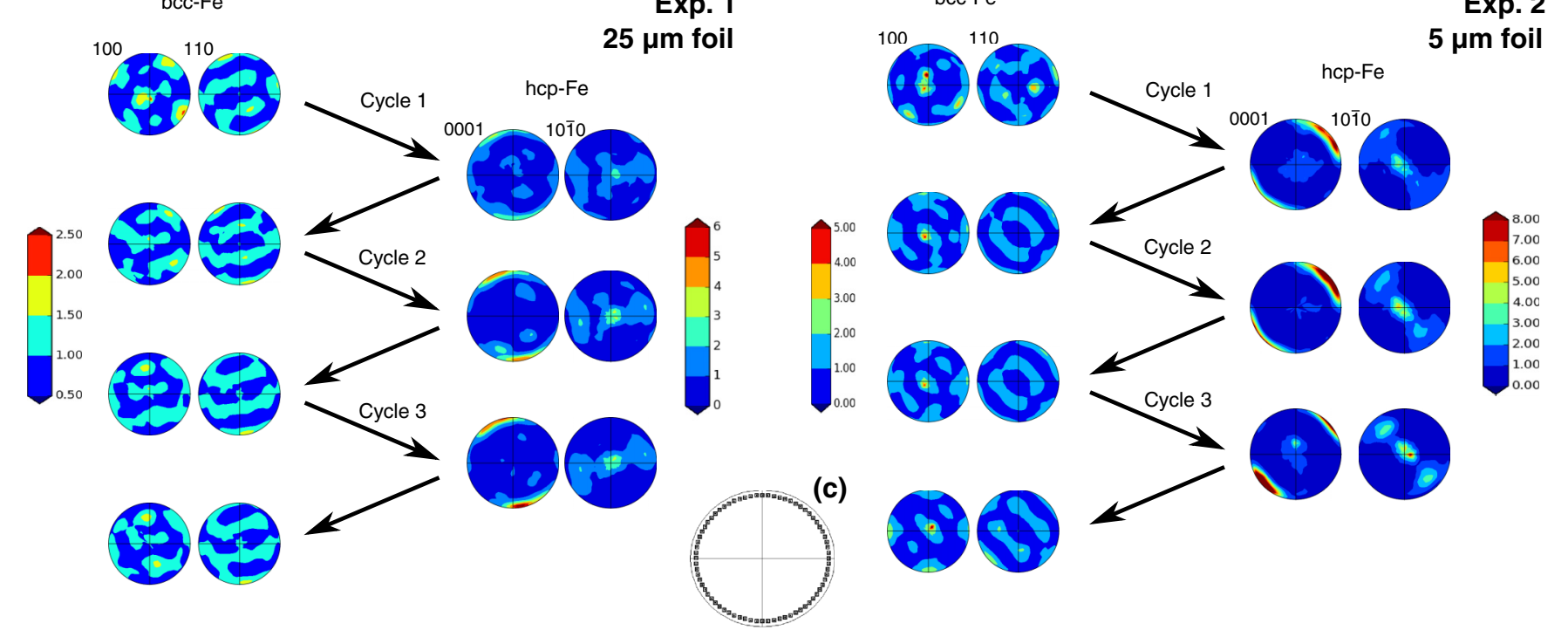

(b)

Exp. 2

$5 \mu \mathrm{m}$ foil

FIG. 7. Pole figures representing orientation statistics induced by the successive $\alpha \leftrightarrow \epsilon$ phase transitions in Fe in Exp. 1 (a) and 2 (b). Left and right color scales indicate texture strength in $\alpha$ - and $\epsilon$-Fe, respectively (scale in multiples of a random distribution, m.r.d.). Texture in $\alpha$-Fe are represented by 110 and 100 pole figures of the compression direction, with the compression direction at the center of the projection. Textures in $\epsilon$-Fe are represented by $10 \overline{1} 0$ and 0001 pole figures of the compression direction. (c) shows the pole figure coverage of the experimental data.
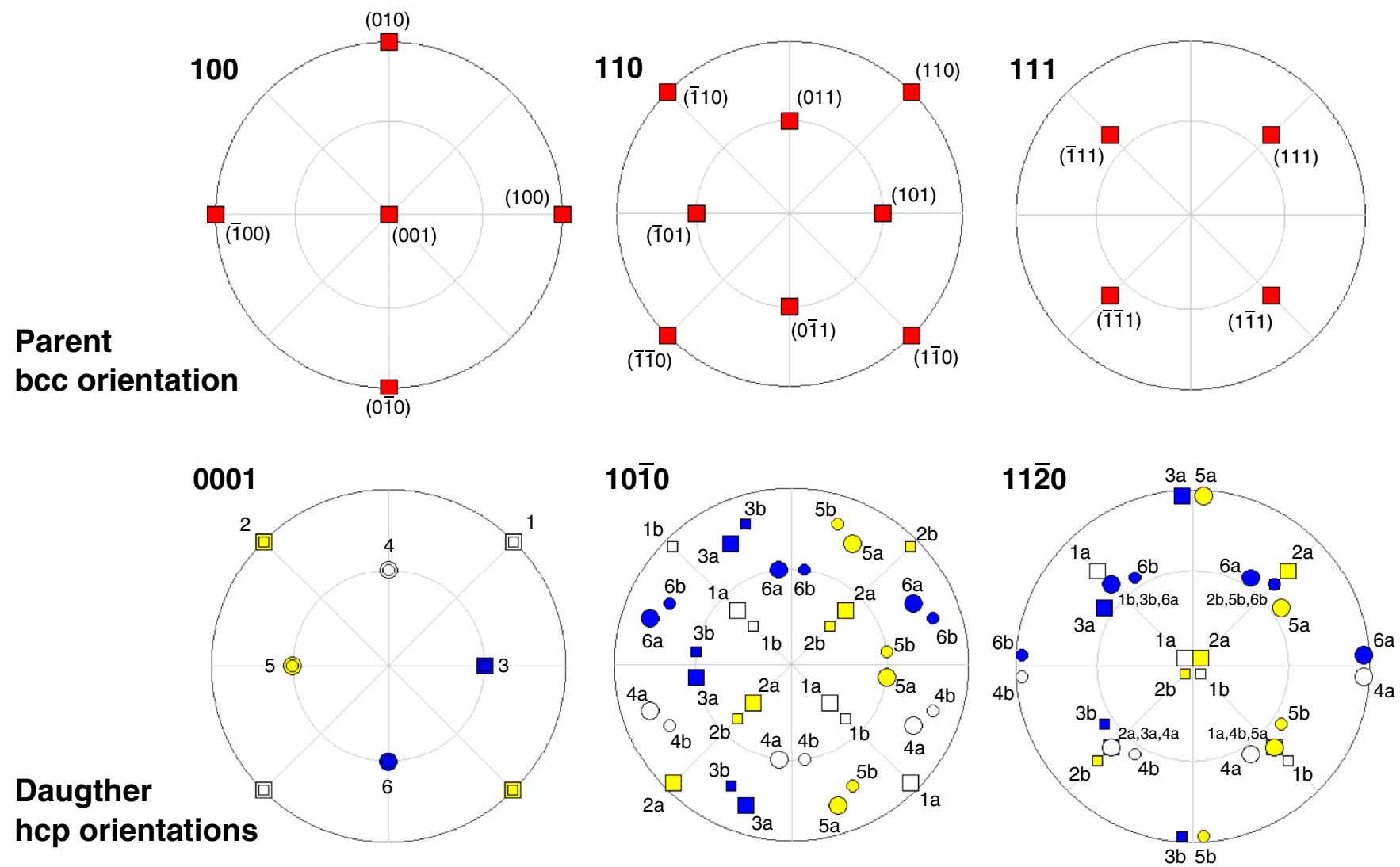

FIG. 8. Orientation of the $12 \epsilon$-Fe variants relative to the parent $\alpha$-Fe orientation according to the Burgers orientation relationships and the notations of Table I. The parent $\alpha$-Fe orientation is used as a reference. The 12 daughter orientations of $\epsilon$-Fe are represented using 0001 , $10 \overline{1} 0$, and $11 \overline{2} 0$ pole figures. 

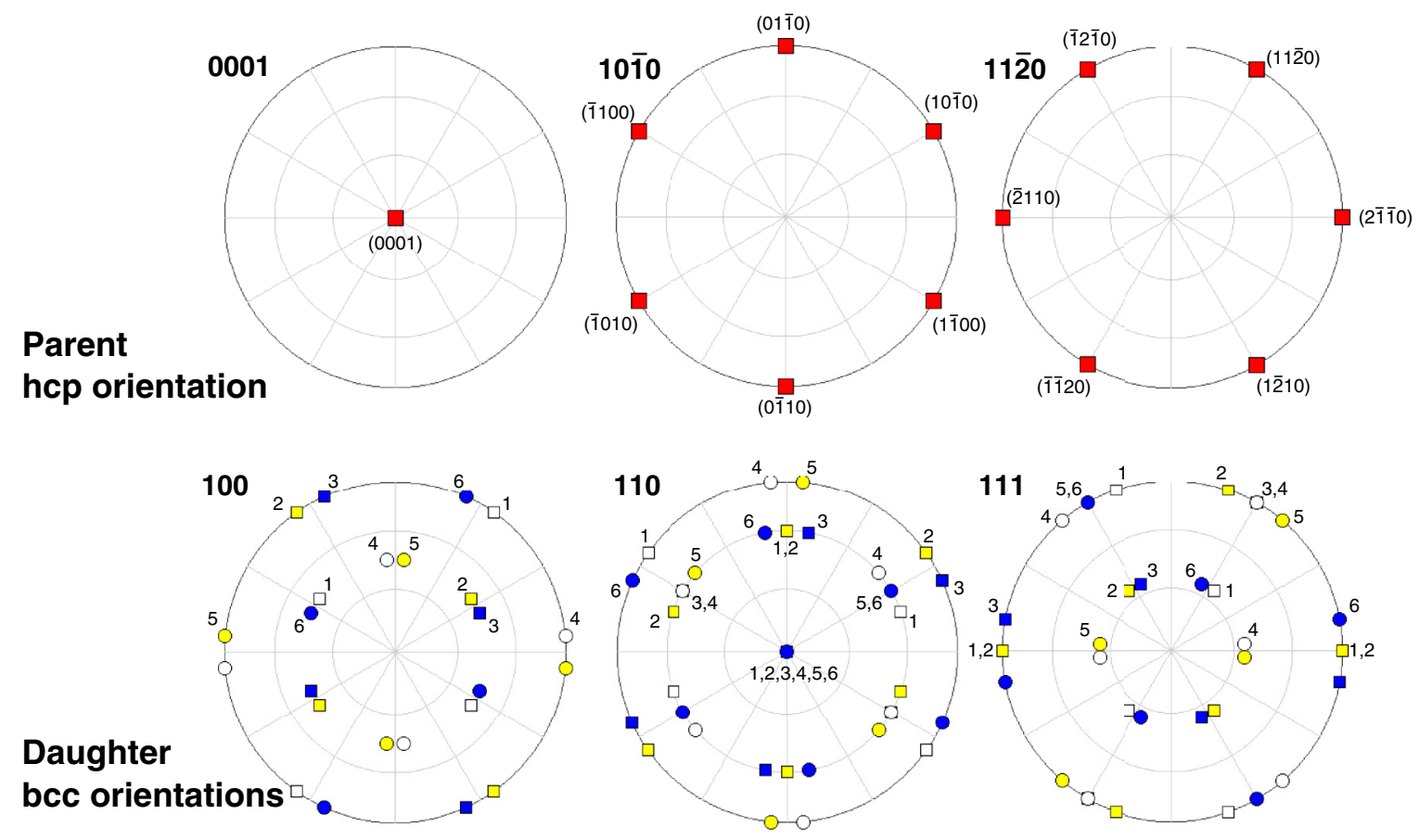

FIG. 9. Orientation of the six $\alpha$-Fe variants relative to the parent $\epsilon$-Fe orientation according to the Burgers orientation relationships and the notations of Table II. The parent $\epsilon$-Fe orientation is used as a reference. The six daughter orientations of $\alpha$-Fe are represented using 100 , 110 , and 111 pole figures.

The correspondence between experimental and simulated $\alpha$-Fe is improved by imposing a $20 \%$ memory effect in the back-transformation, imposing that $20 \%$ of the $\alpha$-Fe domains return to orientations of the original texture. This $20 \%$ memory effect in the $\epsilon$ to $\alpha$ transformation is effective at matching experimental and simulated textures for both samples and at all pressure cycles.

\section{DISCUSSION}

\section{A. Phase transformation hysteresis}

The $\alpha \rightarrow \epsilon$ starts at $14 \pm 1 \mathrm{GPa}$ (Fig. 4) and completes at $18 \pm 1 \mathrm{GPa}$, independent of pressure cycle. This range of transformation pressure is consistent with a previous singlecrystal x-ray diffraction study [33]. Electrical conductivity measurements [2] indicate a much sharper range of transition under hydrostatic conditions while Mössbauer spectroscopy [8] and previous measurements on polycrystals (e.g., Fig. 4 in Ref. [33]) show a much broader range of coexistence between $\alpha$ - and $\epsilon$-Fe. The range of transition pressures between $\alpha$ $\mathrm{Fe}$ and $\epsilon-\mathrm{Fe}$ expands with increasing differential stress [2]. We can hence speculate that the previous polycrystal x-ray diffraction [33] and Mössbauer spectroscopy [8] experiments were performed under higher stress conditions. The electrical conductivity measurements [2] show a smaller pressure transition interval indicative of either lower stress in those

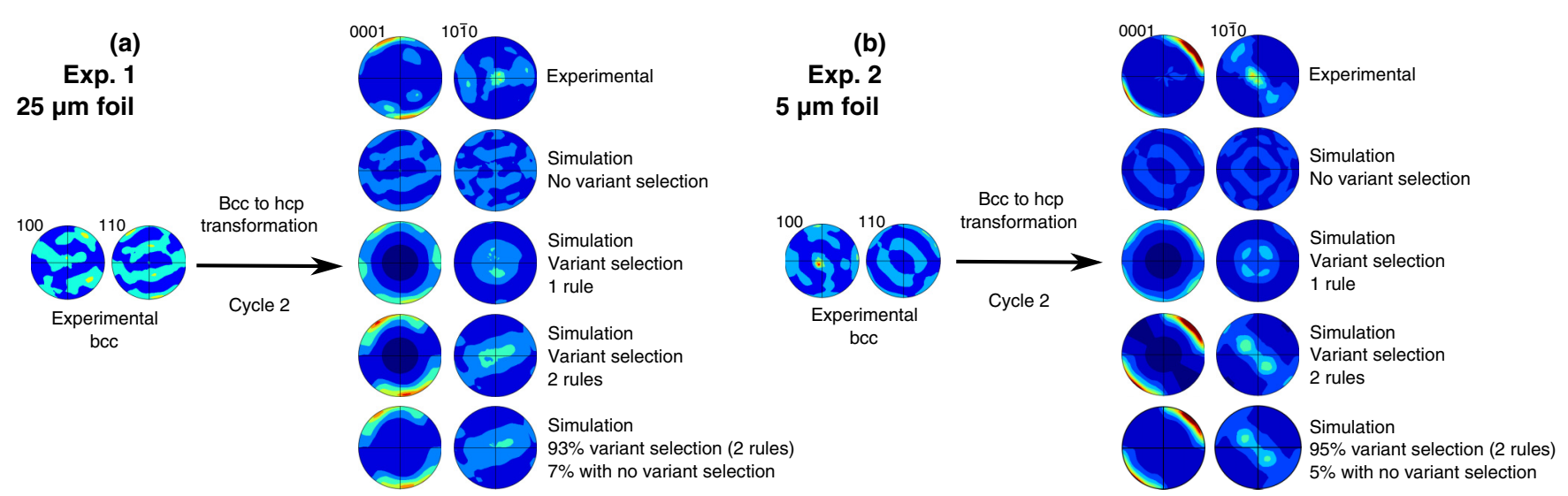

FIG. 10. Sample simulation of the $\alpha$-Fe to $\epsilon$-Fe transformation for Exp. 1 (a) and 2 (b). In both cases, the figure shows the experimental $\alpha-\mathrm{Fe}$ and $\epsilon$-Fe textures along with results of simulations using a full Burgers mechanism (no variant selection) and variant selection rules applied to match the experimental and simulated textures (see text for details). Intensity scales for the pole figures are identical to those of Fig. 7. 
experiments or a different mechanism of transition for electrical conductivity. Indeed, one can expect electrical conductivity to be dependent on the presence or absence of a network of the high-pressure phase rather than a full conversion.

The reverse $\epsilon \rightarrow \alpha$ transformation starts at $10.5 \pm 0.5 \mathrm{GPa}$ and completes at $6 \pm 1 \mathrm{GPa}$, independent of sample or pressure cycle. The onset of the reverse $\epsilon \rightarrow \alpha$ transformation is below previous estimates: 12 GPa using x-ray diffraction [33], $11 \mathrm{GPa}$ using electrical conductivity [2], and over $15 \mathrm{GPa}$ using Mössbauer spectroscopy [8]. Again, phase transitions are affected by differential stress. The lower pressure of transition onset in our experiment and the reproducibility of transition pressures upon increasing cycle numbers probably indicates that, in our experiments on polycrystals, local stress levels were below those of previous works.

\section{B. $c / a$ ratio for $\epsilon-\mathrm{Fe}$}

All our experiments, as well as recent experiments on hcp$\mathrm{Fe}[33,45,46]$, show a smooth evolution of the $c / a$ ratio of $\epsilon$-Fe with pressure (Fig. 6).

The anomalous evolution of $c / a$ ratios of $\epsilon$-Fe measured at the onset of the $\alpha \rightarrow \epsilon$ transition in earlier studies [7,9] is simply not observed. These experiments were performed under nonhydrostatic conditions (i.e., with epoxy pellets as pressure transmitting media in Ref. [9]). After transformation, the $c$ axes of the polycrystal lie perpendicular to compression while $a$ axes are distributed in all directions. The $c / a$ ratio measured in textured and stressed $\epsilon$-Fe will hence be larger than the true $c / a$ ratio of the material.

All recent experiments performed under hydrostatic conditions (i.e., with helium as a pressure medium) simply do not show such anomalous evolution of the $c / a$ ratio of $\epsilon-\mathrm{Fe}$ with pressure.

\section{Transition mechanism between $\alpha$ - and $\epsilon$-Fe}

Both the forward and reverse transformation can be adequately simulated using Burgers ORs. We therefore confirm a Burgers-type atomic path for the $\alpha-\epsilon$ transformation in iron $[15,16,18,23,24,33]$.

Moreover, x-ray diffraction images are well modeled using a mixture of bcc and hcp structures, with no need for introducing an intermediate fcc structure suggested in other studies [9]. Such intermediate fcc phase is not observed in our experiments.

\section{Microstructures induced by the $\alpha \rightarrow \epsilon$ transition}

The forward $\alpha \rightarrow \epsilon$ transformation is heavily affected by variant selection (Figs. 7 and 10). These results are in somewhat disagreement with measurements in single crystals [33] in which all 12 variants of the Burgers OR were reported. These measurements, however, were performed with $(100)_{\alpha}$ perpendicular to compression. In such geometry, all variants of the Burgers OR lead to $c$ axes of $\epsilon$-Fe at $45^{\circ}$ or more to compression. With $(110)_{\alpha}$ perpendicular to compression, one would expect missing transformation variants in $\epsilon$-Fe.

Recent experiments report different textures for $\epsilon$-Fe transformed from $\alpha$-Fe, with observations of both $c$ axes perpendicular and parallel to compression [39]. These measurements are performed above $40 \mathrm{GPa}, 25 \mathrm{GPa}$ above the transformation to $\epsilon$-Fe, and for a sample confined in $\mathrm{SiO}_{2}$, a very stiff pressure-transmitting medium. Plastic deformation is known to align $c$ axes of $\epsilon$-Fe parallel to compression through the action of pyramidal slip of $\langle c+a\rangle$ dislocations and $\{10 \overline{1} 2\}$ tensile twinning [10,52,53]. Measurements of textures with $c$ axes parallel to compression in these experiments can hence be safely be assigned to the effect of plastic deformation.

$\epsilon$-Fe $c$ axes align perpendicular to compression after transformation from $\alpha$-Fe, likely due to nonhydrostatic stresses in the diamond anvil cell experiment. We used helium as a pressure medium for which differential stress should not exceed $0.1 \mathrm{GPa}$ in the current pressure range [40]. We can hence conclude that the $\alpha \rightarrow \epsilon$ transformation is very sensitive with a variant selection driven by the macroscopic stress.

Moreover, $\epsilon$-Fe $c$ axes concentrate a single orientation perpendicular to compression (Fig. 7), in both our experiments and in that of Ref. [39]. Such orientation cannot be explained by the geometry of the experiment and was not observed in experiments on single crystals [33]. One can speculate that, due to internal stresses in the Fe foils, crystallites in the $\mathrm{Fe}$ polycrystal follow a second variant selection rule that favors common variants between adjacent grains.

Overall, the $\alpha \rightarrow \epsilon$ transformation is highly sensitive to both macroscopic and intragranular stresses, inducing variant selection and a strong texture after transformation. This would need to be confirmed and modeled using simulations such as phase-field models [26,38]. One could also consider experiments starting for isolated pure-Fe grains inside a hydrostatic pressure medium rather than a foil to decipher the effects of intragranular and average stresses on the transformation. This goes beyond the scope of the current paper.

\section{E. Microstructures induced by the $\epsilon \rightarrow \alpha$ transition}

As show in Fig. 11, the experimental textures for $\alpha$-Fe after phase transformation lie between those obtained using simulations using Burgers OR and those of $\alpha$-Fe in the previous cycle. Unlike textures for $\epsilon$-Fe, $\alpha$-Fe do not display obvious features that can serve as an anchor for comparing experimental and simulation results. Nevertheless, we obtain satisfactory models of $\epsilon \rightarrow \alpha$ transformation textures using a Burgers OR model with no variant selection with the addition of 5-20\% orientation memory from the original $\alpha$-Fe texture.

Texture memory has been observed in other phase transformations [27,30] and is often associated to interactions among neighboring grains. Interestingly, the texture memory is not complete and the original texture is mostly erased after three cycles of $\alpha \rightarrow \epsilon \rightarrow \alpha$ transformations. As transformation cycles go on, irreversible changes occur in the microstructures that are later fully induced by the phase transformations. This behavior is different from that observed in single crystals [33] in which a nearly full texture memory persisted after multiple phase transformation cycles. Hence, one can speculate that neighboring grain interactions are more important in $\epsilon$-Fe crystallites formed from a $\alpha$-Fe single crystal than a polycrystal. 

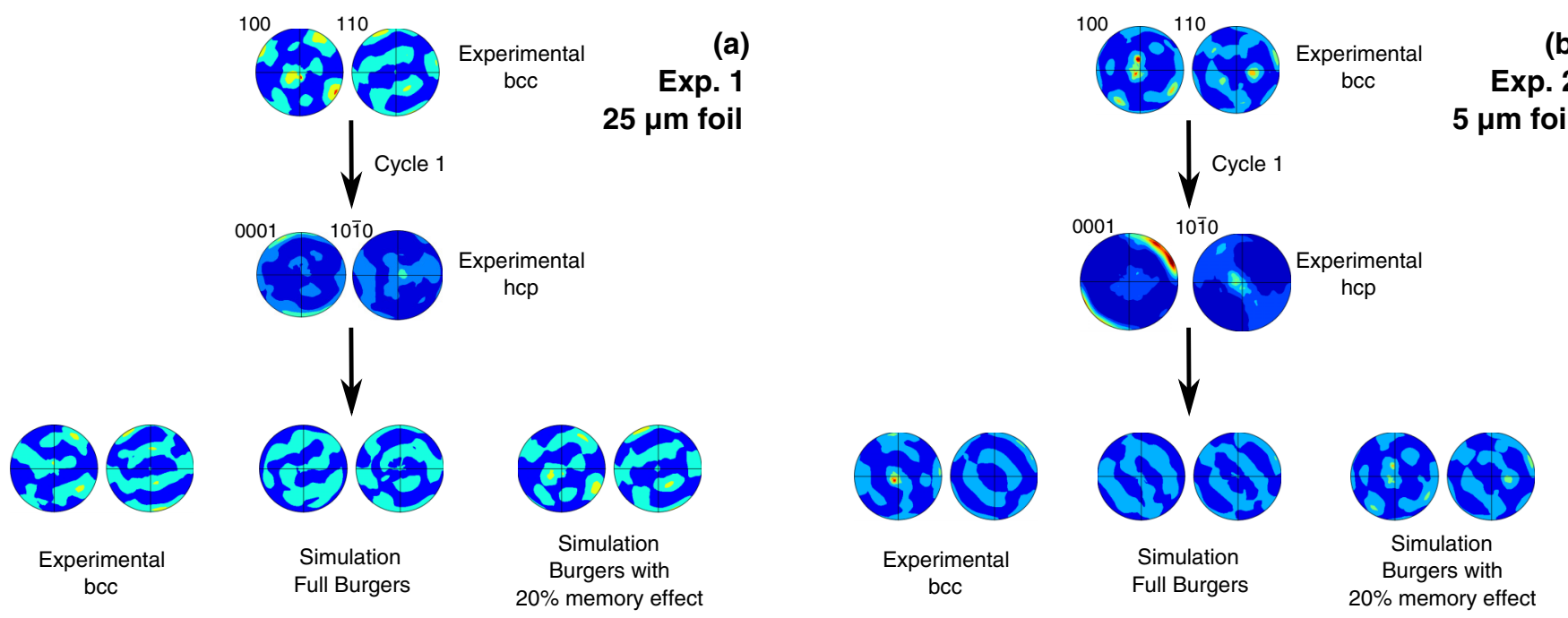

FIG. 11. Sample simulation of the $\epsilon$-Fe to $\alpha$-Fe transformation for Exp. 1 (a) and 2 (b). In both cases, the figure shows the experimental $\alpha$-Fe and $\epsilon$-Fe textures as well as results of simulations using a full Burgers mechanism (no variant selection) and simulations assuming a partial memory effect in the back-transformation to $\alpha$-Fe (see text for details). Intensity scales for the pole figures are identical to those of Fig. 7.

\section{CONCLUSIONS}

This paper focuses on microstructural-effect-induced phase bcc-hcp transformations in Fe polycrystals. The forward $\alpha \rightarrow \epsilon$ transformation in Fe polycrystals under quasihydrostratic conditions starts at $14 \pm 1 \mathrm{GPa}$ and completes at $18 \pm 1 \mathrm{GPa}$ while the reverse $\epsilon \rightarrow \alpha$ transformation starts at $10.5 \pm 0.5 \mathrm{GPa}$ and completes at $6 \pm 1 \mathrm{GPa}$. The average domain size is divided by a factor of 2 during the $\alpha \rightarrow \epsilon$ transformation and returns slightly below those measured at the start of the experiment after the reverse $\epsilon \rightarrow \alpha$ transformation. The $\epsilon-\mathrm{Fe} c / a$ ratio pressure dependence is smooth at all pressure. The anomalous evolutions of $c / a$ ratios of $\epsilon-\mathrm{Fe}$ measured at the onset of the $\alpha \rightarrow \epsilon$ transition in earlier studies is not observed and attributed to large differential stress in early diamond anvil cell experiments.

Both the forward and reverse transformations can be adequately simulated using Burgers ORs. The forward $\alpha \rightarrow \epsilon$ transformation is heavily affected by a variant selection. After transformation, $\epsilon$-Fe crystallites align with their $c$ axes perpendicular to compression, with a concentration of $c$ axes into one single orientation. This is attributed to stress-induced variant selection with two contributions: The differential stress of the diamond anvil cell experiment concentrating $c$ axes perpendicular to compression and the interaction between neighboring grains concentrating $c$ axes into one single orientation. The reverse $\epsilon \rightarrow \alpha$ transformation follows a Burgers path in association with $20 \%$ orientation memory, in contrast with measurements in single crystals for which a nearly full orientation memory is observed.

In the future, such a study could be complete by numerical models of phase transformations [38], including microstructural effects (i.e., grain-to-grain interaction, local stress concentrations, etc.) and average applied stress and deformation. The study could also be completed by experiments on isolated and pure Fe grains. This will allow us to understand the stress dependence and relative effects of macroscopic vs intragranular stress on the $\alpha-\epsilon$ phase transformation in Fe which, to this day, is not clearly understood.

\section{ACKNOWLEDGMENTS}

The authors wish to thank the anonymous reviewers for their constructive comments. All experiments were performed on beamline ID27 at the European Synchrotron Radiation Facility (ESRF), Grenoble, France. We are grateful to the ESRF staff and the ESRF Sample Environment Support Service for providing equipment and assistance in these experiments. The authors also wish to thank A. Dewaële for sharing some of her time at the beamline. This study received support from the Programme National de Planétologie (PNP) of the CNRS, the ARCHI-CM project, and the I-SITE UNLE grant MetalCore (R-ERCGEN-19-006-MERKEL). The project ARCHI-CM of Chevreul Institute (No. FR 2638) is a grant from the Ministère de l'Enseignement Supérieur et de la Recherche, Région Nord-Pas de Calais and European Regional Development Fund (ERDF).
[1] D. Bancroft, E. L. Peterson, and S. Minshall, J. Appl. Phys. 27, 291 (1956).

[2] N. Von Bargen and R. Boehler, High Pressure Res. 6, 133 (1990).
[3] N. Amadou, T. de Resseguier, E. Brambrink, T. Vinci, A. Benuzzi-Mounaix, G. Huser, G. Morard, F. Guyot, K. Miyanishi, N. Ozaki, R. Kodama, and M. Koenig, Phys. Rev. B 93, 214108 (2016). 
[4] J. P. Rueff, M. Krisch, Y. Q. Cai, A. Kaprolat, M. Hanfland, M. Lorenzen, C. Masciovecchio, R. Verbeni, and F. Sette, Phys. Rev. B 60, 14510 (1999).

[5] Q. Wei, C. McCammon, and S. A. Gilder, Geochem. Geophys. Geosyst. 18, 4646 (2017).

[6] H. K. Mao, W. A. Bassett, and T. Takahashi, J. Appl. Phys. 38, 272 (1967).

[7] W. A. Bassett and E. Huang, Science 238, 780 (1987).

[8] R. D. Taylor, M. P. Pasternak, and R. Jeanloz, J. Appl. Phys. 69, 6126 (1991).

[9] F. M. Wang and R. Ingalls, Phys. Rev. B 57, 5647 (1998).

[10] S. Merkel, H.-R. Wenk, P. Gillet, H. K. Mao, and R. J. Hemley, Phys. Earth Planet. Inter. 145, 239 (2004).

[11] O. Mathon, F. Baudelet, J. P. Itie, A. Polian, M. d'Astuto, J. C. Chervin, and S. Pascarelli, Phys. Rev. Lett. 93, 255503 (2004).

[12] A. Monza, A. Meffre, F. Baudelet, J.-P. Rueff, M. d'Astuto, P. Munsch, S. Huotari, S. Lachaize, B. Chaudret, and A. Shukla, Phys. Rev. Lett. 106, 247201 (2011).

[13] N. R. Barton and J. V. Bernier, J. Appl. Cryst. 45, 1145 (2012).

[14] Q. Wei and S. A. Gilder, Geophys Res. Lett. 40, 5131 (2013).

[15] D. H. Kalantar, J. F. Belak, G. W. Collins, J. D. Colvin, H. M. Davies, J. H. Eggert, T. C. Germann, J. Hawreliak, B. L. Holian, K. Kadau, P. S. Lomdahl, H. E. Lorenzana, M. A. Meyers, K. Rosolankova, M. S. Schneider, J. Sheppard, J. S. Stölken, and J. S. Wark, Phys. Rev. Lett. 95, 075502 (2005).

[16] J. Hawreliak, J. D. Colvin, J. H. Eggert, D. H. Kalantar, H. E. Lorenzana, J. S. Stölken, H. M. Davies, T. C. Germann, B. L. Holian, K. Kadau, P. S. Lomdahl, A. Higginbotham, K. Rosolankova, J. Sheppard, and J. S. Wark, Phys. Rev. B 74, 184107 (2006).

[17] L. Dougherty, G. Gray, III, E. Cerreta, R. McCabe, R. Field, and J. Bingert, Scr. Mater. 60, 772 (2009).

[18] S. J. Wang, M. L. Sui, Y. T. Chen, Q. H. Lu, E. Ma, X. Y. Pei, Q. Z. Li, and H. B. Hu, Sci. Rep. 3, 1086 (2013).

[19] Z. Konôpková, A. Rothkirch, A. K. Singh, S. Speziale, and H.-P. Liermann, Phys. Rev. B 91, 144101 (2015).

[20] M. Ekman, B. Sadigh, K. Einarsdotter, and P. Blaha, Phys. Rev. B 58, 5296 (1998).

[21] K. J. Caspersen, A. Lew, M. Ortiz, and E. A. Carter, Phys. Rev. Lett. 93, 115501 (2004).

[22] D. F. Johnson and E. A. Carter, J. Chem. Phys. 128, 104703 (2008).

[23] J. B. Liu and D. D. Johnson, Phys. Rev. B 79, 134113 (2009).

[24] B. Dupé, B. Amadon, Y.-P. Pellegrini, and C. Denoual, Phys. Rev. B 87, 024103 (2013).

[25] N. Gunkelmann, D. R. Tramontina, E. M. Bringa, and H. M. Urbassek, New J. Phys. 16, 093032 (2014).

[26] A. Vattré and C. Denoual, J. Mech. Phys. Solids 92, 1 (2016).

[27] H. R. Wenk, I. Lonardelli, and D. Williams, Acta Mater. 52, 1899 (2004).

[28] M. Daymond, R. Holt, S. Cai, P. Mosbrucker, and S. Vogel, Acta Mater. 58, 4053 (2010).

[29] N. Stanford and P. Bate, Acta Mater. 52, 5215 (2004).
[30] I. Lonardelli, N. Gey, H.-R. Wenk, M. Humbert, S. Vogel, and L. Lutterotti, Acta Mater. 55, 5718 (2007).

[31] C. Cayron, Scripta Mater. 59, 570 (2008).

[32] M. G. Burgers, Physica 1, 561 (1934).

[33] A. Dewaele, C. Denoual, S. Anzellini, F. Occelli, M. Mezouar, P. Cordier, S. Merkel, M. Véron, and E. Rausch, Phys. Rev. B 91, 174105 (2015).

[34] B. Hutchinson, L. Ryde, E. Lindh, and K. Tagashira, Mat. Sci. Eng. A 257, 9 (1998).

[35] C. Jourdan, J. Gastaldi, P. Marzo, and G. Grange, J. Mater. Sci. 26, 4355 (1991).

[36] K. Otsuka and C. M. Wayman (eds.), Shape Memory Materials (Cambridge University Press, Cambridge, UK, 1999).

[37] J. Romero, M. Preuss, and J. Q. da Fonseca, Acta Mater 57, 5501 (2009).

[38] A. Vattré and C. Denoual, J. Mech. Phys. Solids 131, 387 (2019).

[39] K. Ohta, Y. Nishihara, Y. Sato, K. Hirose, T. Yagi, S. I. Kawaguchi, N. Hirao, and Y. Ohishi, Front. Earth Sci. 6, 176 (2018).

[40] S. Klotz, J. C. Chervin, P. Munsch, and G. Le Marchand, J. Phys. D: Appl. Phys. 42, 075413 (2009).

[41] L. Lutterotti, M. Bortolotti, G. Ischia, I. Lonardelli, and H.-R. Wenk, Z. Kristallogr., Suppl. 26, 125 (2007).

[42] W. Kanitpanyacharoen, S. Merkel, L. Miyagi, P. Kaercher, C. N. Tomé, Y. Wang, and H.-R. Wenk, Acta Mater. 60, 430 (2012).

[43] H.-R. Wenk, L. Lutterotti, P. Kaercher, W. Kanitpanyacharoen, L. Miyagi, and R. Vasin, Powder Diffr. 29, 220 (2014).

[44] G. Ischia, H.-R. Wenk, L. Lutterotti, and F. Berberich, J. Appl. Cryst. 38, 377 (2005).

[45] A. Dewaele, P. Loubeyre, F. Occelli, M. Mezouar, P. I. Dorogokupets, and M. Torrent, Phys. Rev. Lett. 97, 215504 (2006).

[46] J. A. Hawreliak, B. El-Dasher, H. Lorenzana, G. Kimminau, A. Higginbotham, B. Nagler, S. M. Vinko, W. J. Murphy, T. Whitcher, J. S. Wark, S. Rothman, and N. Park, Phys. Rev. B 83, 144114 (2011).

[47] U. Dahmen, Acta Metall. 30, 63 (1982).

[48] D. Duly, Acta Metall. Mater. 41, 1559 (1993).

[49] W. Pitsch and A. Schrader, Arch. Eisenhühttenwes. 29, 715 (1958)

[50] D. Potter, J. Less-Common Metals 31, 299 (1973).

[51] W. Rong and G. Dunlop, Acta Metall. 32, 1591 (1984).

[52] H.-R. Wenk, S. Matthies, R. J. Hemley, H. K. Mao, and J. Shu, Nature (London) 405, 1044 (2000).

[53] S. Merkel, M. Gruson, Y. Wang, N. Nishiyama, and C. N. Tomé, Modelling Simul. Mater. Sci. Eng. 20, 024005 (2012).

[54] See Supplemental Material at http://link.aps.org/supplemental/ 10.1103/PhysRevB.102.104103 for the raw data on pressure, phase proportions, cell parameters, and crystallite sizes vs step for both experiments.

[55] A. P. Jephcoat, H. K. Mao, and P. M. Bell, J. Geophys. Res. 91, 4677 (1986). 